\title{
La Influencia de los Niveles de Penetración de la Generación Distribuida en los Mercados Energéticos
}

\author{
Víctor A. Gómez ${ }^{1}$, Cesar Hernández ${ }^{1 *}$ y Edwin Rivas ${ }^{2}$ \\ (1) Facultad Tecnológica, Universidad Distrital Francisco José de Caldas, Bogotá-Colombia. \\ (2) Facultad de Ingeniería, Universidad Distrital Francisco José de Caldas, Bogotá-Colombia. \\ E-mail: victorgomez8807@gmail.com; cahernandezs@udistrital.edu.co; erivas@udistrital.edu.co \\ * Autor a quien debe ser dirigida la correspondencia
}

Recibido Jul. 28, 2017; Aceptado Oct. 6, 2017; Versión final Oct. 30, 2017, Publicado Feb. 2018

\begin{abstract}
Resumen
El presente artículo analiza el modelado de una red de distribución como un mercado energético, pasando de una estructura monopolística a un escenario con un número creciente de competidores. Para lograr este objetivo se evaluó el escenario de una red que hace la transición de la oferta centralizada a distribuida. Se compararon las características del mercado energético, tales como el precio y la cantidad, considerando la generación distribuida y centralizada, y teniendo en cuenta los crecientes niveles de penetración de las energías renovables. Los resultados mostraron que a medida que aumenta el nivel de penetración, el precio de la energía disminuye, aunque muy lentamente debido a la elasticidad precio-demanda de la energía. Finalmente, se concluye que la estructura del mercado de la energía está cambiando debido a las nuevas tecnologías de generación de energía y generación distribuida.
\end{abstract}

Palabras clave: demanda de energía; generación distribuida; mercados energéticos; micro redes

\section{The Influence of Penetration Levels of Distributed Generation in the Energy Markets}

\begin{abstract}
The present paper analyzes the modeling of a distribution network as an energy market, moving from a monopolistic structure to a scenario with an increasing number of competitors. To achieve this objective, the scenario of a network considering the transition from centralized to distributed supply was evaluated. Comparison of the characteristics of the energy market, such as price and quantity, considering the distributed and centralized generation was done, also taking into account the increasing levels of penetration of renewable energies. The results showed that as the level of penetration increases, the price of energy decreases, although very slowly, because of the price-demand elasticity of energy. Finally, it is concluded that the structure of the energy market is changing due to the new technologies of generation of energy and distributed generation.
\end{abstract}

Keywords: energy demand; distributed generation; energy markets; micro grids 


\section{INTRODUCCIÓN}

Las sociedades modernas se enfrentan a una demanda cada vez mayor de energía, lo que implica varios desafíos, entre ellos la escasez prevista de recursos tradicionales de energía fósil y las consecuencias de la contaminación ambiental. El desarrollo de tecnologías de generación de energía y microenergía, tecnologías de generación distribuida (DG, distributed generation) y tecnologías de almacenamiento de energía; requiere que la infraestructura de información de las redes inteligentes (SG, Smart Grid) logren flujos de energía bidireccionales e interacciones de múltiples interesados (Arias et al., 2017; Khan y Arsalan, 2016; Sun y Zhang, 2012). Estos desarrollos están trayendo cambios radicales al modelo tradicional de generación y suministro, así como al modelo de negocios de la industria energética (Houwing et al., 2008; Krajačić et al. 2011; Ren y Ren, 2017) da una descripción y visión de los futuros sistemas energéticos, en donde sus bases estarán en cuatro pilares principales: energías renovables, edificios como centrales de generación y consumo (Ecobuilding), almacenamiento de energía y SG en combinación con vehículos eléctricos (EV, Electric Vehicle) y vehículos a la red (V2G, Vehicle-to-grid). Las inversiones en la generación de energía hidroeléctrica, nuclear, eólica y fotovoltaica ( $P V$, Photovoltaic), así como otras nuevas tecnologías de generación de energía están creciendo continuamente. Sin embargo, el rápido desarrollo de la industria de energía ha revelado algunas debilidades en la seguridad, estabilidad y vulnerabilidad de las redes eléctricas (Houwing et al. 2008).

Una manera eficaz de resolver estos problemas es planificar y desarrollar sistemas de micro redes (MG, Microgrid) en combinación con los sistemas de suministro de energía centralizada tradicionales. En comparación con las redes eléctricas grandes y convencionales, las MG pueden estar compuestas por las cargas y los micro-generadores. Es decir, la DG puede estar contenida en la MG, como la generación de energía eólica o fotovoltaica (Daoutis y Dialynas, 2009; Qamar y Janajreh, 2016). Tanto el emplazamiento como la capacidad de una fuente DG tienen diferentes influencias sobre las corrientes de cortocircuito, las tensiones de bus y los flujos de potencia de las redes de distribución (Gupta y Daratha, 2017; Molina y Mercado, 2010; Rüther y Braun, 2009; Urbanetz et al., 2012). El despliegue de generación distribuida enfrenta varias barreras técnicas a medida que aumenta el nivel de penetración. Es así como la planificación de las redes de distribución de energía con el aumento de la dirección general también requiere cambios adicionales en la política económica. A medida que aumenta el nivel de penetración, se abordan diferentes escenarios económicos y los respectivos resultados que lleva cada implementación.

El presente artículo, el cual se basa en la investigación desarrollada en Vahl et al. (2013), pretende analizar un modelado de una red de distribución como un mercado energético, pasando de una estructura monopolística a un escenario con un número creciente de competidores. Así mismo, se analiza el escenario de una red considerando la transición de la oferta centralizada a distribuida. El presente estudio, el cual se basa en la investigación desarrollada en Vahl et al. (2013) permite evaluar los posibles contextos económicos que puedan aparecer en futuras redes de energía. El objetivo de este artículo es comparar las características del mercado energético, tales como el precio y la cantidad, considerando la generación distribuida y centralizada, teniendo en cuenta también los crecientes niveles de penetración de las energías renovables.

\section{PRINCIPALES ACTORES DE LA GENERACIÓN DE ENERGÍA DISTRIBUIDA}

La DG significa generalmente que una pequeña unidad de generación de energía basada en energía renovable, se encuentra cerca de la carga (Ackermann y Knyazkin, 2002; Zhang X. et al. 2017). Las nuevas formas de energías y/o energías renovables incluyen energía hidroeléctrica, energía eólica, energía solar, energía biológica, energía geotérmica y energía oceánica (Arias et al., 2017; Sun y Zhang, 2012) . En el contexto energético brasileño, la Agencia Nacional de Energía Eléctrica (ANEEL) incluye otras fuentes de energía en un sistema de DG, aun cuando no provengan de fuentes renovables, definiendo a DG en general como generadores de pequeña escala distribuidos a lo largo de la red. La MG es una pequeña red de distribución que está compuesta de unidades de generación, Almacenamiento Distribuido (DS, Distributed Storage), equipo de conversión de energía y sistemas de control. Estas pueden realizar una gestión flexible de la energía (Obara et al., 2011) en un entorno residencial, industrial y comercial.

Las unidades de DG en MG implican una variedad de formas de energía, tales como PV, energía eólica, micro-turbinas de gas y Ciclos Combinados de Calor y Potencia (CCHP, Combined Cooling, heat and Power). Una unidad de DG puede suministrar energía eléctrica a la carga local y alimentar la energía eléctrica sobrante a la red eléctrica. Es así como los sistemas de administración y gestión energética, puede ajustar la energía de salida a usuarios secundarios, de la generación distribuida que satisface las demanda de calefacción y refrigeración de usuarios primarios (Brooks et al., 2017). La diferencia entre las MG y DG, es que las MG pueden funcionar conectadas a la red de alimentación o funcionan independientemente, desconectadas de una red grande en el caso de una falla considerable de la red. Con el fin de eliminar el impacto en las grandes redes eléctricas, es necesario mejorar la estructura de la MG, garantizando los parámetros de configuración y las estrategias de control (García-Triviño et al., 2016). 
Los sistemas de generación de energía renovable son débiles en términos de estabilidad de la fuente de alimentación, ya que las fuentes dependen principalmente de las condiciones climáticas. Sin embargo, algunos de ellos, como la irradiación solar y la velocidad del viento, tienen perfiles complementarios. Por lo tanto, el Sistema de Energía Híbrido Distribuido (DHES, Distributed Hybrid Energy System), compuesto de varias fuentes de generación de energía nueva, es la dirección de desarrollo futuro en los nuevos sistemas de generación de energía de la red eléctrica. EI DHES es un nuevo sistema de suministro de energía que combina energía distribuida y tecnología de almacenamiento de energía distribuida (Kusakana, 2016). Las características de los sistemas híbridos de energía son superar la inestabilidad de un único suministro de energético, lograr la ventaja complementaria y mejorar la eficiencia de diferentes fuentes de energía utilizando diferentes características de varias micro-fuentes, asignar racionalmente micro-fuentes y sistemas de almacenamiento de energía, y al mismo tiempo, mejorar la fiabilidad del suministro de electricidad y la calidad de la alimentación en los estados de funcionamiento de conexión y/o aislamiento mediante estrategias de control de nivelación.

\section{EL MERCADO DE LA ENERGÍA}

El mercado de la energía es distintivo para cada país. Sin embargo, la estructura orgánica se asemeja en los actores cuyas funciones se describen en el campo la generación, trasmisión, comercialización y distribución de la energía eléctrica. Así pues, el conjunto de mercados se estructura en varios niveles tales como: acuerdos privados bilaterales fuera del mercado de valores, mercado diario o al contado, intra-díarios en tiempo real. Entre estos, el mercado spot, en donde el activo financiero es pagado al contado al momento de la entrega del servicio (comúnmente conocido como el mercado al contado en un corto periodo de tiempo), es uno de los más relevantes ya que un $30 \%$ del consumo energético de la Unión Europea (UE) se basa a través de este modelo de mercado.

El mercado spot de energía permite a los productores de suministro eléctrico localizar instantáneamente a los compradores disponibles y brindar el excedente del servicio energético que puede suplir las necesidades de la demanda. Igualmente, este mercado permite negociar los precios en milisegundos y entregar energía al cliente unos minutos más tarde. Los mercados spot pueden ser operados privadamente o controlados por organizaciones industriales o agencias gubernamentales (De Bragança y Daglish, 2016). La lógica microeconómica detrás del diseño de los mercados europeos de energía spot es que los generadores más eficientes reciben un ingreso adicional, por encima de su costo marginal. De esta manera se apropian de la diferencia entre el precio de mercado, determinado por el costo marginal de generar menos eficientemente y sus propios costos marginales; a través de este ingreso adicional, pueden cubrir sus costos fijos y remunerar el capital invertido (Rubin y Babcock, 2011). Este ingreso adicional es también un signo económico para las nuevas inversiones: las plantas más eficientes logran ganancias extraordinarias, por encima de lo que normalmente se espera para la industria.

Sin embargo, en Cramton y Stoft, (2005), este modelo tiene tres problemas distintos, que tienden a ser evitados a través de la intervención del regulador. El primer problema se refiere a la señal económica para la inversión, representada por la posible ganancia de nuevos generadores eficientes. Esta señal económica no funciona adecuadamente para inversiones en plantas de capital altamente intensivas como la nuclear o la hidroeléctrica, por ejemplo. Los proyectos de este tipo son muy difíciles de reproducir sin contratos a largo plazo que garanticen un alto nivel de ingresos predecibles y fijos. Dado que el precio de referencia es el precio al contado, los consumidores y proveedores no están motivados a asumir voluntariamente el riesgo de soportar un posible desajuste entre los costos de los contratos a largo plazo y los precios de mercado.

La segunda distorsión que el diseño del mercado se centró en un mercado spot diario puede referirse al comportamiento de los precios en picos de demanda (Harris, 2006). En tales situaciones, la carga es suministrada por generadores con mayores costos variables. Dado que la demanda de electricidad es inelástica a corto plazo, estos generadores, que son los últimos en la orden de despacho, tienen el poder de exigir precios mucho más altos que sus costos marginales (Guthrie y Videbeck, 2007). Como los precios más altos se consideran socialmente difíciles de justificar, el regulador a menudo establece un límite para el precio spot. A pesar de ello, el modelo de negocio para los generadores se utiliza esporádicamente y por lo tanto, no se ve comprometido. Por el contrario, los generadores deben tener, en algunas situaciones, ingresos sobre costos variables para remunerar la inversión y otros costos fijos. En consecuencia, para resolver este problema, las políticas energéticas deben incluir regulaciones relativas a la remuneración de la energía, capaces de permitir este modelo de negocio, al tiempo que garantizan la fiabilidad del sistema durante la demanda máxima.

La tercera cuestión es la dificultad de tratar adecuadamente la creciente participación de la generación renovable en la combinación (Liu et al., 2016). El aumento de la generación de fuentes renovables tiene el efecto de disminuir la cuota de mercado de los generadores alimentados con combustibles fósiles. La 
generación de energía a partir de fuentes renovables tiende a presentar estructuras de costos centradas en costos fijos, inversiones altas y costos marginales muy bajos o casi nulos. Contradictoriamente, los autores en Milstein y Tishler (2011) afirman que una gran reducción en los costos de las tecnologías PV podría aumentar la adopción de esta alternativa energética, pero también puede aumentar el precio promedio de la energía y la volatilidad durante los períodos en que las condiciones climáticas limitan su potencia de salida de estas tecnologías.

\section{MODELADO DE UN MERCADO DE LA ENERGÍA DG}

Como se señala en Cutler et al. (2011) y Liu et al. (2016), una gran reducción en la capacidad renovable aumenta la adopción de los costos, pero también creará nuevos desafíos para el mercado de la energía. Por lo tanto, al considerar la promoción de las energías renovables para reducir las emisiones de dióxido de carbono (CO2), los reguladores deberían evaluar el comportamiento del mercado de la electricidad, particularmente con respecto a las características de las tecnologías renovables, así como las incertidumbres de la demanda y el suministro. Con base en lo anterior, en Genc y Sen (2008) estudiaron la evolución de las inversiones de capital y el comportamiento de precios de los proveedores relacionados con la demanda de electricidad, utilizando herramientas de programación estocástica. Concluyendo que, en un mercado de tres jugadores, la capacidad de instalación total y los precios de mercado son más altos que en un mercado de cinco jugadores. Por consiguiente, en la siguiente sección se presentará el modelo propuesto en Vahl et al. (2013) con el objetivo de describir la influencia de la penetración de la DG en la estructura económica.

Los autores en Baiocchi y Capelo (1984), Nagurney et al. (2007) y Noor et al. (1993) presentan varios problemas complejos de equilibrio de red usando desigualdades variacionales. Aunque no están relacionados con el mercado de la energía, ellos discuten el modelado de problemas reales y aplicaciones usando esta teoría. Por ejemplo, en Nagurney et al. (2007) desarrollaron un modelo de equilibrio de la red de la cadena de suministro de energía eléctrica estática con demandas conocidas, estableciendo la equivalencia entre su modelo y un modelo de equilibrio de red de transporte con demandas fijas.

Los problemas de optimización no limitados y restringidos pueden ser formulados como problemas de desigualdad variacional, aunque este último puede formularse como un problema de optimización, sólo cuando la condición de simetría y la condición de semi-definición positiva se mantienen. Por lo tanto, la desigualdad variacional es el problema más general que también puede manejar una función $f(x)$ con un Jacobiano asimétrico, superando la asunción de simetría de muchos problemas de equilibrio que se han formulado como problemas de optimización, que es restrictivo en términos de aplicaciones y más realista en el modelado de materias primas, modos y/o clases en competencia (Nagurney et al., 2007).

La oferta y la demanda son conceptos fundamentales de la economía. En un mercado competitivo, los principios básicos de estos dos pilares determinan el precio y la cantidad vendida de bienes y servicios. De igual manera, la relación oferta $y$ demanda del mercado energético está regido por estos principios económicos (ver Fig. 1). Por ejemplo, un cierto patrón de producción, transporte y consumo de un producto determinado, denotado por $S^{*}, Q^{*}, d^{*} \in K$, está en equilibrio sólo si satisface el problema de desigualdad variacional, demostrado en Noor et al. (1993) y expuesto en Vahl et al. (2013), a través de la ecuación (1).

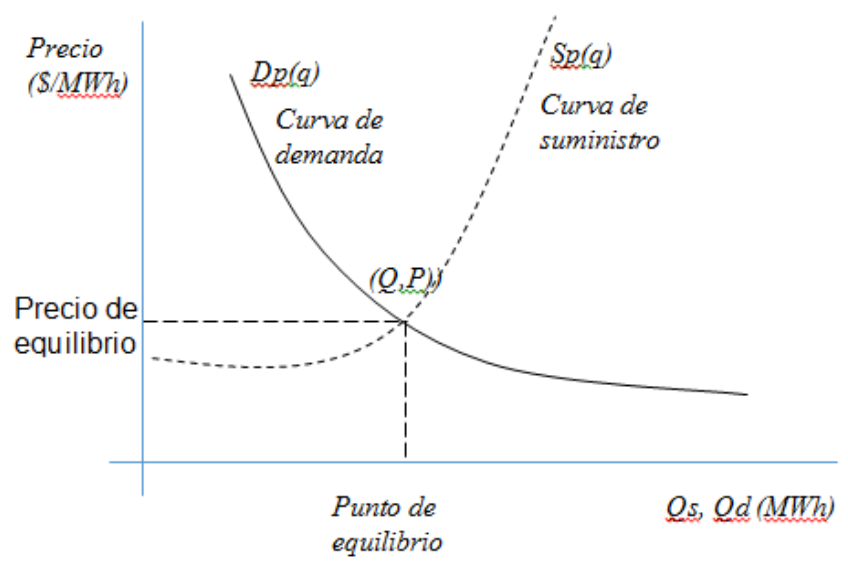

Fig. 1: Curvas de oferta y demanda energética (Vahl et al., 2013)

El modelo más sencillo que describe la Fig. 1, se relaciona en las tendencia del consumo en función del precio del servicio y la venta del mismo. Asumiendo que en un mercado de competencia perfecta, el precio de generación eléctrica será establecido en el punto de equilibrio en donde cada kW que se genera es adoptado sin el precepto de una demanda no satisfecha. 


$$
\left(\left[\begin{array}{c}
\pi\left(s^{*}\right) \\
C\left(\mathrm{Q}^{*}\right) \\
-\rho\left(d^{*}\right)
\end{array}\right],\left[\begin{array}{c}
s \\
\mathrm{Q} \\
d
\end{array}\right]-\left[\begin{array}{c}
s^{*} \\
\mathrm{Q}^{*} \\
d^{*}
\end{array}\right]\right) \geq 0 ; \forall(\mathrm{s}, \mathrm{Q}, \mathrm{d}) \in \mathrm{K}
$$

En donde $\pi\left(S^{*}\right)$ es la función de producción; $C\left(Q^{*}\right)$ es la función de transporte y $\rho\left(d^{*}\right)$ es la función de consumo.

En cuanto a la generación de energía distribuida, también podemos identificar los mismos parámetros del mercado, ya que la energía es realmente una mercancía. Sin embargo, la estructura del mercado energético es variable por la desregulación y las nuevas políticas. Es así, como la generación centralizada de energía es considerada un mercado monopolístico puesto que unos pocos productores proveen el servicio, dadas las necesidades del sector. Sin embargo, si aumenta el nivel de la DG, incluso si comienza con sólo unos pocos productores de potencia, el mercado se convierte en un oligopolio, en donde la participación de un pequeño número de proveedores garantice la suplencia del servicio. Es así como los niveles más altos de penetración de la DG pueden lograr un mercado completamente libre y/o de libre competencia.

Cada estructura de mercado requiere un enfoque adecuado. Por ejemplo, el modelo de equilibrio demostrado en Cournot y Fisher (1897), J. F. Nash (1950) y J. Nash (1951) establece que un mercado oligopolístico dado alcanza el equilibrio, ya que cada productor que participa en el oligopolio tiene el objetivo de maximizar su propio beneficio (ver ecuación (2)), como un problema de optimización que permite determinar los valores de las variables que intervienen en el sector energético para luego obtener el máximo beneficio de las partes involucradas.

$$
u i(x)=\sum_{j=1}^{n} P_{j}\left(d_{j}\right) x_{i j}-\sum_{j=1}^{n} C_{i j}\left(X_{i j}\right) x_{i j}-\int_{0}^{s_{i}} \pi_{i}(w) d w
$$

En la ecuación (2), x es la cantidad transportada y consumida; $X_{i j}$ es la cantidad transportada desde el proveedor $i$ al mercado $j ; Z_{i}$ es el beneficio del proveedor $i$; $D_{j}$ es la ecuación de la curva de demanda para el mercado $j$ (variando de 1 a N); $\pi_{i}(w)$ es la ecuación de oferta (costo) para el proveedor $i ; C_{i j}$ es el costo de transporte de llevar las mercancías del i-ésimo proveedor al j-ésimo mercado.

Un vector de estrategia dado $X^{*}$ es un equilibrio de las ecuaciones presentadas en Griffin y Schulman (2005) y Vahl et al. (2013) si y sólo si, $X^{*} \in$ k es una solución para la desigualdad variacional (ver ecuación (3)).

$$
\left\langle F\left(\mathrm{x}^{*}\right), \mathrm{x}-\mathrm{x}^{*}\right\rangle \forall x \in K
$$

Donde $F(x) \equiv\left(-\nabla_{x 1} u 1(x), \ldots,-\nabla_{x m} u_{m}(x)\right)$ es un vector lineal.

El primer termino está orientado a encontrar el equilibrio de dicho mercado oligopolístico. Es necesario encontrar la formulación adecuada para aplicarla en la desigualdad variacional. Por lo tanto, el problema de optimización se transforma en la ecuación (4).

$$
\frac{\partial u_{i}(x)}{\partial x_{i j}}=\rho_{j}\left(d_{j}\right)+\frac{\partial}{\partial d_{j}} \rho_{j}\left(d_{j}\right) x_{i j}-C_{i j}\left(x_{i j}\right)-\pi_{i}\left(s_{i}\right)
$$

El beneficio de cada generador distribuido es entonces una consecuencia del equilibrio del mercado de la energía, y es también, una consecuencia de la estructura del mercado. El equilibrio se mantiene cuando la función derivada mostrada en la ecuación (4) es cero. Para obtener más información y los algoritmos sobre cómo resolver este problema ver las referencias presentadas en Vahl et al. (2013).

\section{FUNCIÓN DE DEMANDA DE ENERGÍA}

La teoría económica básica dice que a medida que suban los precios de la energía, la cantidad de energía demandada caerá, manteniendo todos los demás factores constantes. Sin embargo, los estudios sugieren que la demanda de energía de los consumidores es menos sensible a los cambios de precios que la demanda 
de muchos otros productos básicos (Nieto, 2016). La relación entre la demanda de energía y el precio es pequeña; la demanda de energía es relativamente inelástica al precio (Behboodi et al., 2016; Bentzen y Engsted, 1993; Bernstein y Griffin, 2006; Zhang et al., 2017). También hay diferencias en la elasticidad a corto y largo plazo para la demanda de energía, así como en diferentes lugares, incluso dentro de un país (Griffin y Schulman, 2005). También se ha demostrado que existen diferencias de elasticidad entre los sectores como se evidencia en Behboodi et al., (2016) y Labandeira et al.,(2017).

La elasticidad de precios puede usarse para interpretar cómo la demanda de los consumidores responde a los cambios de precios, la facilidad con que los consumidores pueden comprar sustitutos para un producto que ha subido de precio o incluso cuánto valoran los consumidores un bien en particular. La elasticidad de los precios puede utilizarse de esta manera debido a la teoría subyacente de la respuesta del consumidor a los cambios de precios: un consumidor con un presupuesto fijo a corto plazo tiene tres posibles respuestas a un cambio de precio (Bohi y Zimmerman, 1984): en primer lugar, el consumidor puede comprar otro bien como sustituto; en segundo lugar el consumidor puede comprar menos del bien sin la correspondiente compra de un sustituto; y tercero el consumidor puede continuar comprando la misma cantidad del bien y reducir gastos en otros bienes.

En el caso de la electricidad y otros mercados de suministro de energía, estos productos tienen un grado limitado de ser sustituidos, especialmente a corto plazo. Sin embargo, considerando la DG como un sustituto adecuado de la energía de la red eléctrica, los consumidores pueden comenzar a enfrentar un dilema diferente a partir de ahora. Estudios futuros sobre los mercados de energía donde la DG está presente pueden actualizar este escenario. A pesar de estas cuestiones, encontrar la relación entre el precio de la energía y la demanda es crucial para el análisis de equilibrio de un mercado. Por ejemplo, la ecuación (5) muestra la función de demanda derivada (Cobb Douglas) para la energía eléctrica, la cual ha sido linealizada (Behboodi et al., 2016; Bernstein y Griffin, 2006; Irffi et al., 2009).

$$
\log D_{t}=\log k+\alpha \log P_{t}+\beta \log Y_{t}+\delta \log L_{t}+\phi \log S_{t}
$$

Aquí, $D_{t}$ es la demanda de energía durante el tiempo $t ; P_{t}$ es el precio de la energía; $Y_{t}$ es la renta (tasa media para el residencial y el PIB para el comercio y la industria); $L_{t}$ es el precio de los aparatos electrónicos (en cada sector); $S_{t}$ es el precio sustitutivo de la energía de las empresas eléctricas (esto se utiliza principalmente para el sector industrial, pero la DG también puede ofrecer una oferta alternativa para otros sectores); $\alpha$ es la elasticidad precio-demanda; $\beta$ es la demanda de elasticidad-ingreso; y $\delta$ es la elasticidad de la demanda debido a los precios de los electrodomésticos.

\section{DG y los costos marginales}

La función de costo de suministro para los proveedores de energía depende principalmente de los costos operativos y de mantenimiento de las tecnologías. La Tabla 1 muestra los costos estimados para las diferentes fuentes no convencionales de energía para Colombia. Los cuales detallan la implementación de tecnologías con una línea base de tiempo estimada entre los 20 y 30 años (UPME, 2015). No obstante, es importante conocer los costos marginales de transmisión y distribución locales, junto con los costos marginales de energía, que constituyen el costo marginal total contra el cual se debe evaluar la eficiencia energética y la DG (Huang y Söder, 2017).

Estudios como éste pueden proporcionar datos para evaluar la penetración y el desarrollo de la DG en países que aún no han introducido las tecnologías de la DG o en aquellos donde su plan de expansión contempla la implementación de Recursos Energéticos Distribuidos (DER, Distributed Energy Resources) para zonas no interconectadas.

\section{Costos y pérdidas de distribución}

Las pérdidas de distribución se evalúan considerando las pérdidas de potencia en la línea desde el nodo generador hasta el nodo consumidor. Más precisamente, se puede considerar el uso de las pérdidas del generador al nodo de distribución y de ahí al medidor de energía. Como se consideran los nodos para evaluar el equilibrio del mercado, se toman solamente los costos de nodo a nodo.

Como se necesita encontrar una relación de pérdida de energía por distancia y energía transmitida, se propone estimar un coeficiente basado en la pérdida nominal máxima. Sea el cable 2 AWG, el cable de recorrido estudiado, con una intensidad máxima (Imax) de 138 A a 13,8 MVA y una resistencia lineal ( $r$ ) de $0,86 \Omega$ por kilómetro. La pérdida de potencia máxima por distancia, considerando solo el efecto de joule y factor de potencia unitario, se puede calcular de acuerdo con la ecuación (6). 
Tabla 1: Resumen de costos O\&M en energías alternativas (UPME, 2015)

\begin{tabular}{|c|c|c|c|}
\hline Ítem & Unidad & Valor & Tecnología \\
\hline \multirow[t]{5}{*}{ Cargos O\&M fijos } & \multirow[t]{5}{*}{ USD/MW } & 6.500 & Para la tecnología solar \\
\hline & & 40.000 & Para la tecnología eólica \\
\hline & & 87.000 & Para geotérmica \\
\hline & & 21.000 & Para biomasa solida (caña de azúcar) \\
\hline & & 40.000 & Para biogás derivado de efluentes de palma de aceite \\
\hline \multirow[t]{2}{*}{ Cargos O\&M variables } & USD/MW & - & Para tecnología solar, eólica y geotérmica \\
\hline & USD/MWh & 11,5 & Para biomasa sólida y biogás \\
\hline Cargos CND y ASIC & USD/MW & 0,33 & Solo aplica para empresas de servicios públicos \\
\hline Ley 99 de 1993 & $\begin{array}{l}\text { \% de la venta } \\
\text { de energía }\end{array}$ & 4 & $\begin{array}{l}\text { Para plantas mayores a } 10 \mathrm{MW} \text { cuya producción de energía } \\
\text { se base en procesos térmicos }\end{array}$ \\
\hline Ley 99 de 1993 & USD/MWh & 31,62 & Precio de energía para aplicación ley 99/93 \\
\hline Ley 143 de 1994 & $\begin{array}{l}\% \text { de los gatos } \\
\text { operacionales }\end{array}$ & $1 \%$ & Solo aplica para empresas de servicios públicos (Utilities) \\
\hline
\end{tabular}

$$
P_{\text {loss }}\left(\frac{W}{k m}\right)=I_{\max }^{2}(A) \cdot \mathrm{r}\left(\frac{\Omega}{k m}\right)=138^{2}(0.86)=15,23(\mathrm{~kW} / \mathrm{km})
$$

Es importante hace notar que en la ecuación (6), el valor de la resistencia será un valor único. Sin embargo, una aproximación más detallada debe considerar la variación de la temperatura y la naturaleza de cada material.

Por lo tanto, teniendo en cuenta la necesidad del análisis de mercado de convertir dichas pérdidas a valores monetarios, también tenemos que multiplicar el coeficiente de pérdida de potencia $\left(P_{\text {loss }}\right)$ de la línea eléctrica utilizada, por la longitud de línea desde el nodo del proveedor al consumidor, y el precio de la energía en el mercado consumidor presentado en la ecuación (7).

$$
P_{l o s s}(\$)=P_{l o s s} \cdot x_{i j} \cdot p_{j}=4,6\left(\frac{k W}{M W h} \cdot k m\right) \cdot p_{j} \cdot x_{i j}
$$

La función de costo de la pérdida de energía en cada generador-consumidor de trayectoria se obtiene usando la ecuación (7). El resultado es el costo promedio del cable por MWh. Este último sería el costo de transporte de la energía.

Durante el cálculo del equilibrio, dicho costo puede variarse de acuerdo con el foco de interés; después de todas las iteraciones del algoritmo tenemos los costos totales de transmisión y distribución, debido a las pérdidas de potencia en las líneas, de acuerdo con la energía total comercializada a lo largo de los caminos. Esta evaluación por sí sola ya es útil para diseñar sistemas de transmisión y distribución de energía y localizar generadores, ya que diferentes caminos entre el generador y el consumidor presentarán diferentes pérdidas de potencia, por lo que distinguirán eficiencias del sistema. Estos costos pueden atribuirse al consumidor y son conocidos como Costo Libre Abordo (FOB, Free On Board) que son atribuidos al cliente por pérdidas en el transporte de energía. Por otro lado, tenemos los Costos de Seguro y Flete (CIF, Insurance and Freight) que son atribuidos a los trámites arancelarios a los que debe optar el proveedor cuando desea comercializar el suministro energético. Estos dos costos son tratados en los acuerdos comerciales entre ambas partes.

Los argumentos expuestos presentan la influencia de las principales variables a tener en cuenta en la eficiencia de los sistemas energéticos partiendo de los análisis técnico-económicos. Por ejemplo, con la ecuación (7) se detalló como la influencia de los generadores y las respectivas líneas de distribución, interactúan en todo momento en la relación de costos entre el consumidor y el proveedor.

\section{Beneficios de las DG}

Sobre la base de los resultados expuestos anteriormente, los beneficios que la DG brinda a los mercados energéticos pueden enumerarse de la siguiente manera: (1) aumenta el tamaño del mercado dando la posibilidad a que otros actores participen en la cadena de valor energética; (2) disminuye el flujo de energía 
de la red eléctrica o proveedor convencional que se encuentra en una red centralizada; (3) los costos se ven reducidos debido a la disminución en las pérdidas del sistema; (4) se obtienen una variación en los precios de la energía acorde a la elasticidad precio de la demanda; (5) de garantizarse la oferta energética a través de dispositivos de almacenamiento, se lograría garantizar un sistema con mayores prestaciones en relación a la confiabilidad y la continuidad del servicio; (6) es una importante aproximación de los costos que pueden tener los usuarios conectados a una red descentralizada o distribuida.

\section{Desafíos en la implementación de las DG}

Dentro de los desafíos en la implementación de las DG, se encuentran los siguientes: (1) de no contar con tecnologías de almacenamiento acordes a la demanda máxima del sistema, el sistema será susceptibles a variaciones en la calidad de la potencia eléctrica. (2) Es importante que la DG tenga un abanico de posibilidades en las tecnologías de generación, esto permitirá disminuir la probabilidad de que exista deficiencia en el servicio y por ende una fluctuación de los precios. (3) La libre competencia debe garantizar la conformación de oligopolios en donde la eficiencia del sistema garantice la comercialización de la energía a precios razonables. (4) Es importante establecer una política de precios de las actividades de operación y mantenimiento, igualmente crear la posibilidad de incentivar la mejora en la prestación del servicio y la continua evidencia de los planes de mejora continua e inversión que propicien los proveedores del servicio energético. (5) Es importante que se realicen los respectivos estudios de viabilidad que permitan tener un panorama de la eficiencia que pudiera tener un sistema. Por ejemplo, en la implementación de un sistema solar fotovoltaico, se esperaría realizar de manera previa un estudio del histórico de radiación del área geográfica en donde se implementará tal tecnología. (6) Es importante que el desarrollo de cada proyecto contemple los estudios, técnicos, económicos, culturales y ambientales que permitan tener en el radar todas las variables que inciden en la implementación de proyectos a partir de DG.

\section{DISCUSION FINAL}

La estructura del mercado de la energía está cambiando debido a las nuevas tecnologías de generación de energía y la llamada DG. El principal objetivo en este artículo fue mostrar a partir de un estudio previo, cómo la penetración de la DG puede interferir en la estructura del mercado a partir de las desigualdades y variacionales presentes y teniendo en cuenta la transición de la generación centralizada a un nivel de penetración del $100 \%$ en fuentes no convencionales de suministro. Los resultados mostraron que a medida que aumenta el nivel de penetración, se supone que el precio de la energía disminuye, aunque muy lentamente debido a la elasticidad precio-demanda de la energía.

Los sistemas de generación de energía fotovoltaica y eólica alcanzarán una cuota de mercado considerable, ya que son tecnologías que presentan una creciente viabilidad económica para sustituir las tecnologías de generación convencionales por sólidas estructuras de mercado a niveles maduros y, por tanto, precios energéticos más bajos que el suministro centralizado. Sin embargo, las cuestiones técnicas, como la intermitencia de la generación, requieren el respaldo de seguridad en formas de energía de otras fuentes. Por lo tanto, a menos que estén asegurados por los sistemas de almacenamiento o la red de servicios convencionales, los niveles de penetración del 100\% en DG no son sostenibles para los generadores fotovoltaicos y eólicos, incluso para los sistemas híbridos que combinan ambos. El almacenamiento de energía es entonces estratégico para aumentar los niveles de penetración de tecnologías eólicas y PV, manteniendo la confiabilidad del sistema. Además, estos sistemas de almacenamiento pueden aumentar el retorno de la inversión en las DG, suministrando energía a horas de máxima demanda, cuando se alcanzan precios más altos.

Por último, la planificación de políticas energéticas nacionales trae nuevos dilemas con la introducción de la DG. La teoría económica sugiere que un mercado perfectamente competitivo conduciría a una fijación de precios eficiente. En ausencia de competencia, los reguladores desempeñan un papel fundamental para atraer financiamiento a precios razonables a fin de mantener, renovar y aumentar la infraestructura y prestar servicios a un costo razonable. El equilibrio de los precios del mercado de la energía depende principalmente de los proveedores, generadores, fuentes de energía y de la demanda, representados por los usuarios de la red de servicios públicos convencionales.

\section{CONCLUSIONES}

De acuerdo con el trabajo presentado y los resultados obtenidos, se pueden plantear las siguientes conclusiones principales:

El principal objetivo en este artículo fue mostrar a partir de un estudio previo, cómo la penetración de la generación distribuida puede interferir en la estructura del mercado a partir de las desigualdades y variacionales presentes y teniendo en cuenta la transición de la generación centralizada a un nivel de 
penetración del $100 \%$ en fuentes no convencionales de suministro. Los resultados mostraron que a medida que aumenta el nivel de penetración, el precio de la energía disminuye, aunque muy lentamente debido a la elasticidad precio-demanda de la energía.

Pese a los costos de operación y mantenimiento, países como Colombia alcanzarán una cuota de mercado considerable en generación de energía fotovoltaica y eólica, ya que son tecnologías que presentan una creciente viabilidad económica para sustituir las tecnologías de generación convencionales por sólidas estructuras de mercado a niveles maduros y, por tanto, precios energéticos más bajos que el suministro centralizado.

Por último, la planificación de políticas energéticas nacionales trae nuevos dilemas con la introducción de la generación distribuida. La teoría económica sugiere que un mercado perfectamente competitivo conduciría a una fijación de precios eficiente. En ausencia de competencia, los reguladores desempeñan un papel fundamental para atraer financiamiento a precios razonables a fin de mantener, renovar y aumentar la infraestructura y prestar servicios a un costo razonable. El equilibrio de los precios del mercado de la energía depende principalmente de los proveedores, generadores, fuentes de energía y de la demanda, representados por los usuarios de la red de servicios públicos convencionales.

\section{AGRADECIMIENTOS}

Los autores de este artículo desean agradecer a Colciencias y a la Universidad Distrital Francisco José de Caldas por la financiación de los recursos para el desarrollo de este proyecto de investigación.

\section{REFERENCIAS}

Arias, L.; Rivas, E. y León, L., Propuesta de modelo de Gestión para redes eléctricas con Generación Distribuida a través de Unidades de Medición Fasorial. Información Tecnológica, 28(2), 15-28 (2017).

Ackermann, T. y V. Knyazkin, Interaction between distributed generation and the distribution network: operation aspects, IEEE/PES Transmission and Distribution Conference and Exhibition, 1357-1362, Yokohama, Japan, October 6-10 (2002).

Arias, L.; E. Rivas y L. León, Propuesta de modelo de Gestión para redes eléctricas con Generación Distribuida a través de Unidades de Medición Fasorial. Información Tecnológica, 28(2), 15-28 (2017).

Baiocchi, C. y A.C. Capelo, Variational and quasivariational inequalities: Applications to free boundary problems, 1-462, John Wiley \& Sons, New York, USA, (1984).

Behboodi, S.; D.P. Chassin; Crawford, C., y Djilali, N., Renewable resources portfolio optimization in the presence of demand response. Applied Energy, 162, 139-148 (2016).

Bentzen, J. y T. Engsted, Short-and long-run elasticities in energy demand: a cointegration approach. Energy Economics, 15(1), 9-16 (1993).

Bernstein, M. A. y J. M. Griffin, Regional differences in the price-elasticity of demand for energy. Citeseer (2006).

Bohi, D.R., y M. B. Zimmerman, An update on econometric studies of energy demand behavior. Annual Review of Energy, 9(1), 105-154 (1984).

Brooks, B.; J. Jiang y H. Sun, Incorporating user utility in a smart microgrid with distributed generation and elastic demand, IEEE International Conference on Communications Workshops, 1117-1122, Paris, Francia, Mayo 21-25 (2017).

Cournot, A. A. y I. Fisher, Researches into the Mathematical Principles of the Theory of Wealth. Macmillan Co (1897).

Cramton, P. y S. Stoft, A capacity market that makes sense. The Electricity Journal, 18(7), 43-54 (2005).

Cutler, N.J.; N.D. Boerema; I.F. MacGill y H.R. Outhred, High penetration wind generation impacts on spot prices in the Australian national electricity market. Energy Policy, 39(10), 5939-5949 (2011).

Daoutis, L.G. y E.N. Dialynas, Impact of hybrid wind and hydroelectric power generation on the operational performance of isolated power systems. Electric Power Systems Research, 79(10), 1360-1373 (2009).

De Bragança, G.G.F. y T. Daglish, Can market power in the electricity spot market translate into market power in the hedge market? Energy Economics, 58, 11-26 (2016). 
García-Triviño, P.; J.P. Torreglosa; L.M. Fernández-Ramírez y F. Jurado, Control and operation of power sources in a medium-voltage direct-current microgrid for an electric vehicle fast charging station with a photovoltaic and a battery energy storage system. Energy, 115, 38-48 (2016).

Genc, T.S. y S. Sen, An analysis of capacity and price trajectories for the Ontario electricity market using dynamic Nash equilibrium under uncertainty. Energy Economics, 30(1), 173-191 (2008).

Griffin, J. M. y C. T. Schulman, Price asymmetry in energy demand models: a proxy for energy-saving technical change? The Energy Journal, 1-21 (2005).

Gupta, N. y N. Daratha, Probabilistic three-phase load flow for unbalanced electrical systems with wind farms. International Journal of Electrical Power \& Energy Systems, 87, 154-165 (2017).

Guthrie, G. y S. Videbeck, Electricity spot price dynamics: Beyond financial models. Energy Policy, 35(11), 5614-5621 (2007).

Harris, P.G., Relationship between Competitive Power Markets and Grid Reliability: The PJM RTO Experience. Recommendation, 12, 87 (2006).

Houwing, M.; A.N. Ajah; P.W. Heijnen; I. Bouwmans y P.M. Herder, Uncertainties in the design and operation of distributed energy resources: the case of micro-CHP systems. Energy, 33(10), 1518-1536 (2008).

Huang, Y. y L. Söder, Evaluation of economic regulation in distribution systems with distributed generation. Energy, 126, 192-201 (2017).

Irffi, G.; I. Castela; M.L. Siqueira y F.C. Linhares, Previsão da demanda por energia elétrica para classes de consumo na região Nordeste, usando OLS dinâmico e mudança de regime. Econ. Aplicada, 13(1), 6998 (2009).

Khan, J. y M. H. Arsalan, Solar power technologies for sustainable electricity generation-A review. Renewable and Sustainable Energy Reviews, 55, 414-425 (2016).

Krajačić, G.; N. Duić; A. Tsikalakis; M. Zoulias; G. Caralis; E. Panteri y M. Graça Carvalho, Feed-in tariffs for promotion of energy storage technologies. Energy Policy, 39(3), 1410-1425 (2011).

Kusakana, K. Optimal scheduling for distributed hybrid system with pumped hydro storage. Energy Conversion and Management, 111, 253-260 (2016).

Labandeira, X.; J.M. Labeaga y X. López-Otero, A meta-analysis on the price elasticity of energy demand. Energy Policy, 102, 549-568 (2017).

Liu, C.; N. Li y D. Zha, On the impact of FIT policies on renewable energy investment: Based on the solar power support policies in China's power market. Renewable Energy, 94, 251-267 (2016).

Milstein, I., y A. Tishler, Intermittently renewable energy, optimal capacity mix and prices in a deregulated electricity market. Energy Policy, 39(7), 3922-3927 (2011).

Molina, M.G., y P.E. Mercado, Stabilization and control of tie-line power flow of microgrid including wind generation by distributed energy storage. International Journal of Hydrogen Energy, 35(11), 5827-5833 (2010).

Nagurney, A.; Z. Liu; M.G. Cojocaru y P. Daniele, Dynamic electric power supply chains and transportation networks: An evolutionary variational inequality formulation. Transportation Research Part E: Logistics and Transportation Review, 43(5), 624-646 (2007).

Nash, J. F., Equilibrium points in n-person games. Proceedings of the National Academy of Sciences, 36(1), 48-49 (1950).

Nash, J., Non-cooperative games. Annals of Mathematics, 286-295 (1951).

Nieto, A., Optimizing prices for small-scale distributed generation resources: a review of principles and design elements. The Electricity Journal, 29(3), 31-41 (2016).

Noor, M. A.; y K. I. Noor y T. M. Rassias, Some aspects of variational inequalities. Journal of Computational and Applied Mathematics, 47(3), 285-312 (1993). 
Obara, S.; S. Watanabe y B. Rengarajan, Operation planning of an independent microgrid for cold regions by the distribution of fuel cells and water electrolyzers using a genetic algorithm. International Journal of Hydrogen Energy, 36(22), 14295-14308 (2011).

Qamar, S. B., y I. Janajreh, Renewable Energy Sources for Isolated Self-sufficient Microgrids: Comparison of Solar and Wind Energy for UAE. Energy Procedia, 103, 413-418 (2016).

Ren, J. y X. Ren, Sustainability Ranking of Energy Storage Technologies under Uncertainties. Journal of Cleaner Production (2017).

Rubin, O.D., y B.A. Babcock, A novel approach for modeling deregulated electricity markets. Energy Policy, 39(5), 2711-2721 (2011).

Rüther, R. y P. Braun, Energetic contribution potential of building-integrated photovoltaics on airports in warm climates. Solar Energy, 83(10), 1923-1931 (2009).

Sun, Z. y X. Zhang, Advances on distributed generation technology. Energy Procedia, 17, 32-38 (2012).

UPME., Integración de las energías renovables no convencionales en Colombia. Bogota (2015).

Urbanetz, J.; P. Braun y R. Rüther, Power quality analysis of grid-connected solar photovoltaic generators in Brazil. Energy Conversion and Management, 64, 8-14 (2012).

Vahl, F.P.; R. Rüther y N. Casarotto Filho, The influence of distributed generation penetration levels on energy markets. Energy Policy, 62, 226-235 (2013).

Zhang, C.; Y. Xu; Z.Y. Dong y K.P. Wong, Robust Coordination of Distributed Generation and Price-Based Demand Response in Microgrids. IEEE Transactions on Smart Grid (2017).

Zhang, X.; H. Chen; Y. Xu; W. Li; F. He; H. Guo y Y. Huang, Distributed generation with energy storage systems: A case study. Applied Energy, 204, 1251-1263 (2017). 
